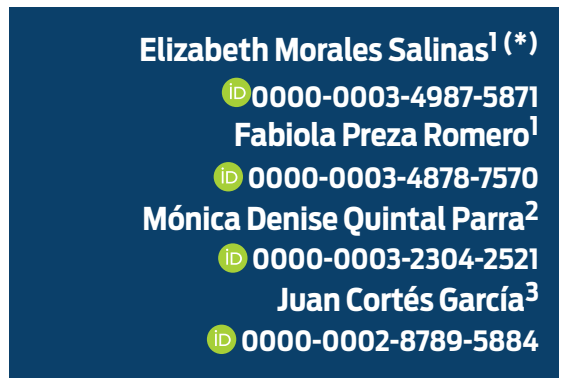

IDepartamento de Patología, Facultad de Medicina Veterinaria y Zootecnia, Universidad Nacional Autónoma de México. Av. Ciudad Universitaria 3000, Col. Copilco Universidad, Delegación Coyoacán, Ciudad de México, CP. 04360.

2 Diagnóstico Privado-HistoVet Yucatán. Calle 20 No. 412, Col. Nueva Alemán, Mérida, Yucatán, México, CP. 97146.

${ }^{3}$ Hospital de Especialidades Veterinarias San Jerónimo. Avenida San Jerónimo 1431. Col. San Jerónimo Lídice,

Delegación Magdalena Contreras, Ciudad de México, CP. 10200.

* Autor para correspondencia: Correo electrónico: moraless@unam.mx

\section{Torsión uterina e hidrometra unilateral en una coneja (Oryctolagus cuniculus) de compañía}

\section{Resumen}

Descripción del caso. Coneja de compañía (Oryctolagus cuniculus) de cinco años de edad, de raza palomino, nulípara, que dejó de comer y beber gradualmente, y estaba apática.

Hallazgos clínicos. Tenía el abdomen notablemente distendido, con peloteo y dolor a la palpación, estaba deshidratada y deprimida. El hemograma y la bioquímica sanguínea indicaron anemia severa altamente regenerativa normocrómica, hiperazotemia prerrenal, hipoproteinemia e hipoalbuminemia. En el ultrasonido y estudio radiográfico, se observó que el útero estaba muy dilatado con abundante líquido, lo que sugería hidrometra, o bien, hemometra.

Tratamiento y evolución. Durante su hospitalización fue medicada con enrofloxacina, tramadol y meloxicam. Al realizar la ovariohisterectomía, se observó que el cuerno uterino derecho, estaba torcido por completo con abundante líquido sanguinolento. Se diagnosticó torsión uterina e hidrometra unilateral derecha. La coneja murió un día después de la cirugía.

Pruebas de laboratorio. En el examen histológico, el endometrio estaba muy hemorrágico y necrosado con pérdida de la mucosa y de las glándulas endometriales, con marcada congestión y edema en todas las capas uterinas. El diagnóstico histopatológico del útero fue emitido como hemorragia y necrosis endometrial grave, difusa y edema transmural grave difuso.
Recibido: 2018-10-02 Aceptado: 2019-02-22 Publicado: 2019-03-28 Información y declaraciones adicionales en la página 11

(c) Derechos de autor: Elizabeth Morales Salinas et al. 2019 acceso abierto

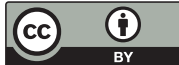

Una forma de citar este artículo:

Morales-Salinas E, Preza-Romero F, Quintal-Parra MD, Cortés-García J. Torsión uterina e hidrometra unilateral en una coneja (Oryctolagus cuniculus) de compañía. Clínica Veterinaria: abordaje diagnóstico y terapéutico. 2019;5:e34201952. DOI: 10.22201/ fmvz.23958766e.201934 
Relevancia clínica. Las enfermedades del tracto reproductor en conejas de edad avanzada y no esterilizadas como el adenocarcinoma uterino y la hiperplasia endometrial son comunes. Estas patologías pueden acompañarse de hidrometra y hemometra afectando a ambos cuernos uterinos. El hallazgo relevante en este caso es que la torsión y la hidrometra de un solo cuerno uterino se presentaron juntas, quizá estaban relacionadas, lo que se ha documentado poco. Las patologías uterinas en conejas se pueden prevenir realizado ovariohisterectomía a edad temprana. Cuando una coneja presenta distención abdominal, además de considerar patologías uterinas como en este caso, se debe descartar enfermedad cardiaca, renal o alguna neoplasia abdominal.

Palabras clave: útero, pseudogestación, cirugía, lagomorfo, patología.

\section{Unilateral uterine torsion and hydrometra in a pet rabbit}

\section{Abstract}

Case description. A five years old, palomino breed, nulliparous, pet female rabbit (Oryctolagus cuniculus), which gradually stop eating and drinking and was apathetic.

Diagnostics and interpretation. Physical examination revealed prominent abdominal distention, pain and puddle sing on abdominal palpation and depression. Hematological and chemistry values revealed severe strongly regenerative normochromic anemia, prerenal azotemia, hypoproteinemia, and hypoalbuminemia. The ultrasound and radiographic study revealed that the uterus was extremely dilated with abundant fluid suggesting hydrometra or hemometra.

Treatment and evolution. The rabbit during her hospitalization was treated with enrofloxacin, tramadol and meloxicam. At ovariohysterectomy, it was observed that the right uterine horn was completely twisted and approximately $800 \mathrm{~mL}$ of bloody fluid were collected from inside it. Unilateral uterine torsion and hydrometra were diagnosed. The rabbit died one day after surgery.

Laboratory tests. Histologically, the endometrium was severely hemorrhagic and necrotic with loss of the uterine epithelium and endometrial glands, in addition there was marked congestion and edema in all the uterine layers. The histopathological diagnosis of the uterus was emitted as severe diffuse hemorrhage and necrosis endometrial and severe diffuse transmural edema.

Discussion and clinical relevance. Reproductive tract diseases in unsterilized mature age rabbits such as uterine adenocarcinoma and endometrial hyperplasia are common. These abnormalities can be accompanied by hydrometra and hemometra affecting both uterine horns. As a relevant finding 
in this case is that the torsion and hydrometra of a single uterine horn were presented together and there may be a relationship between them which has been slight referenced. Uterine pathologies in rabbits can be prevented by performing ovariohysterectomy in young animals. When a female rabbit presents abdominal distention, in addition to considering uterine abnormalities as in this case, heart or kidney disease or some abdominal neoplasm should be included in the differential diagnosis.

Keywords: uterus, pseudopregnancy, surgery, lagomorph, pathology. 


\section{Descripción del caso}

Coneja (Oryctolagus cuniculus) de compañía de cinco años de edad, de 3.7 kg, raza palomino, nulípara y sin antecedentes de otras enfermedades. La coneja vivía dentro de la casa de la dueña, la mitad del día permanecía en su jaula y el resto del día estaba libre. Era alimentada con alimento comercial para conejo (pellets), lechuga, plátano y manzana, y consumía agua ad libitum. La dueña informa que a partir de los dos años de edad, durante tres a cuatro días cada seis meses aproximadamente, tenía comportamiento materno al querer hacer nidos y arrancarse el pelo. La coneja fue remitida a una clínica veterinaria debido a que se encontraba apática y a que, aproximadamente durante tres días antes de la consulta, disminuyó gradualmente el consumo de alimento y agua.

\section{Hallazgos clínicos e interpretación}

Desde el primer día de consulta, la coneja fue hospitalizada. En la inspección física, se notó que el abdomen estaba notablemente distendido, manifestó dolor abdominal, depresión, deshidratación, emaciación, signo de peloteo en abdomen y mucosas ligeramente ictéricas. Se realizó un hemograma y una bioquímica sanguínea de perfil completo. Los resultados más significativos se resumen en los Cuadros 1 y 2.

Los resultados del hemograma se interpretaron de la siguiente manera: anemia severa altamente regenerativa normocrómica. La morfología de eritrocitos mostró esferocitos $1+$, anisocitosis y policromasia $2+$, lo que se asoció a proceso oxidativo (Cuadro 1). ${ }^{1}$

Los resultados del perfil bioquímico se interpretaron de la siguiente manera: hiperazotemia prerrenal por posible deshidratación o insuficiencia renal (no se pudo corroborar ya que no se realizó la necropsia), AST (aspartato aminotransferasa) elevada por esfuerzo y catabolismo muscular e hipoproteinemia por hipoalbuminemia por posible insuficiencia renal por pérdidas a terceros espacios (Cuadro 2). ${ }^{1-2}$

\section{Estudio radiográfico y ultrasonido de abdomen}

El estudio radiográfico mostró aumento generalizado del abdomen, en donde se observa un órgano sugerente de útero que desplaza a las vísceras abdominales hacia craneal (Figuras 1 y 2). En el ultrasonido de abdomen, se observó un órgano con contenido líquido que se presumió era el útero con diagnóstico de probable piometra o hidrometra (Figura 3), linfonodos mesentéricos aumentados de tamaño y lodo biliar.

\section{Tratamiento y evolución}

El primer día de hospitalización la paciente fue medicada con enrofloxacina vía oral, $5 \mathrm{mg} / \mathrm{kg}$ cada 24 h (Rx Spectrum enrofloxacina, Holland de México, SA de CV), ${ }^{3}$ tramadol vía oral, $10 \mathrm{mg} / \mathrm{kg}$ cada $24 \mathrm{~h}$ (Pisadol, Pisa Agropecuaria, SA de CV) y meloxicam vía oral, $0.2 \mathrm{mg} / \mathrm{kg}$ cada 24 h (Sindol, MederiLab, SA de CV). ${ }^{4}$ 
Cuadro 1. Hemograma

\begin{tabular}{|l|c|c|c|}
\hline \multicolumn{1}{|c|}{ Analito } & Resultados & Unidades & Valores derefierencia \\
\hline Hematocrito & 0.14 & $\mathrm{~L} / \mathrm{L}$ & $\mathbf{0 . 2 8}-0.48^{1}$ \\
\hline Hemoglobina & 47 & $\mathrm{~g} / \mathrm{L}$ & $89.63-153.2^{1}$ \\
\hline Eritrocitos & 2 & $\times 10^{12} / \mathrm{L}$ & $4.37-7.43^{1}$ \\
\hline VGM calculado & 70 & $\mathrm{fL}$ & $59.3-69.6^{1}$ \\
\hline Reticulocitos & 332 & $\times 10^{9} / \mathrm{L}$ & - \\
\hline
\end{tabular}

Otros hallazgos: hemólisis ligera, lipemia 2+.

Cuadro 2. Perfil bioquímico

\begin{tabular}{|c|c|c|c|}
\hline Analito & Resultados & Unidades & Valores derefierencia \\
\hline Urea & 21.1 & $\mathrm{mmo} / \mathrm{L}$ & $2.63-10.28^{1}$ \\
\hline AST & 123 & $\mathrm{U} / \mathrm{L}$ & $3.75-32.4^{1}$ \\
\hline Proteínas totales & 48 & $\mathrm{~g} / \mathrm{L}$ & $48.9-73.9^{2}$ \\
\hline Albúmina & 24 & $\mathrm{~g} / \mathrm{L}$ & $35.6-56.8^{2}$ \\
\hline
\end{tabular}

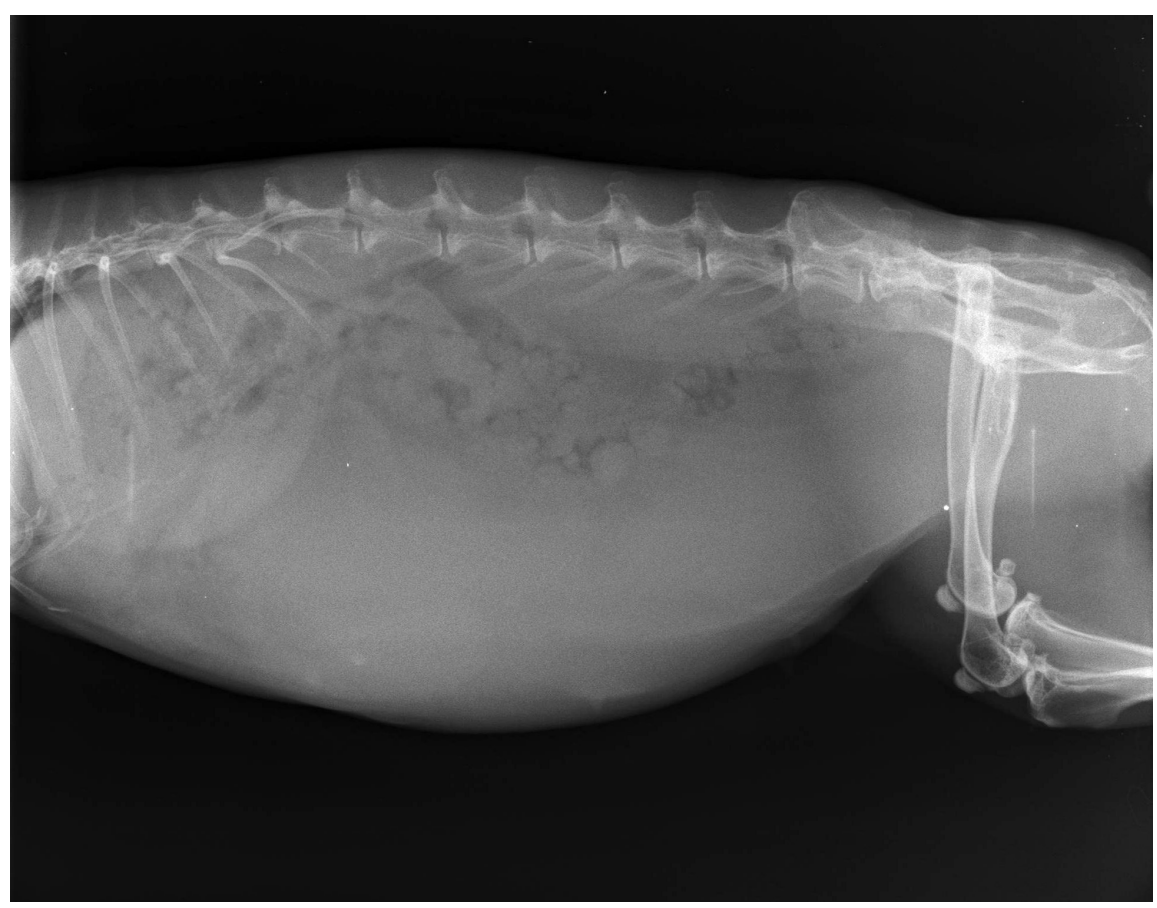

Figura 1. Radiografía laterolateral de abdomen que muestra distención del útero. 


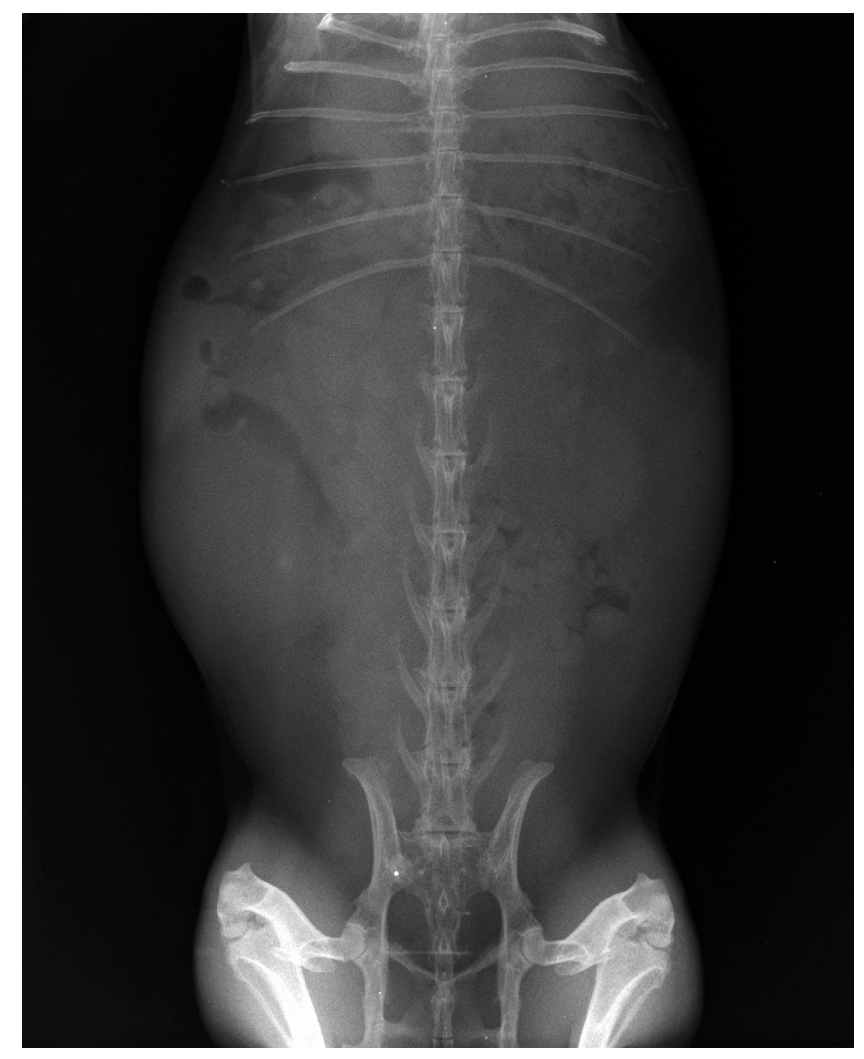

Figura 2. Radiografía ventrodorsal de abdomen que muestra distención del útero.

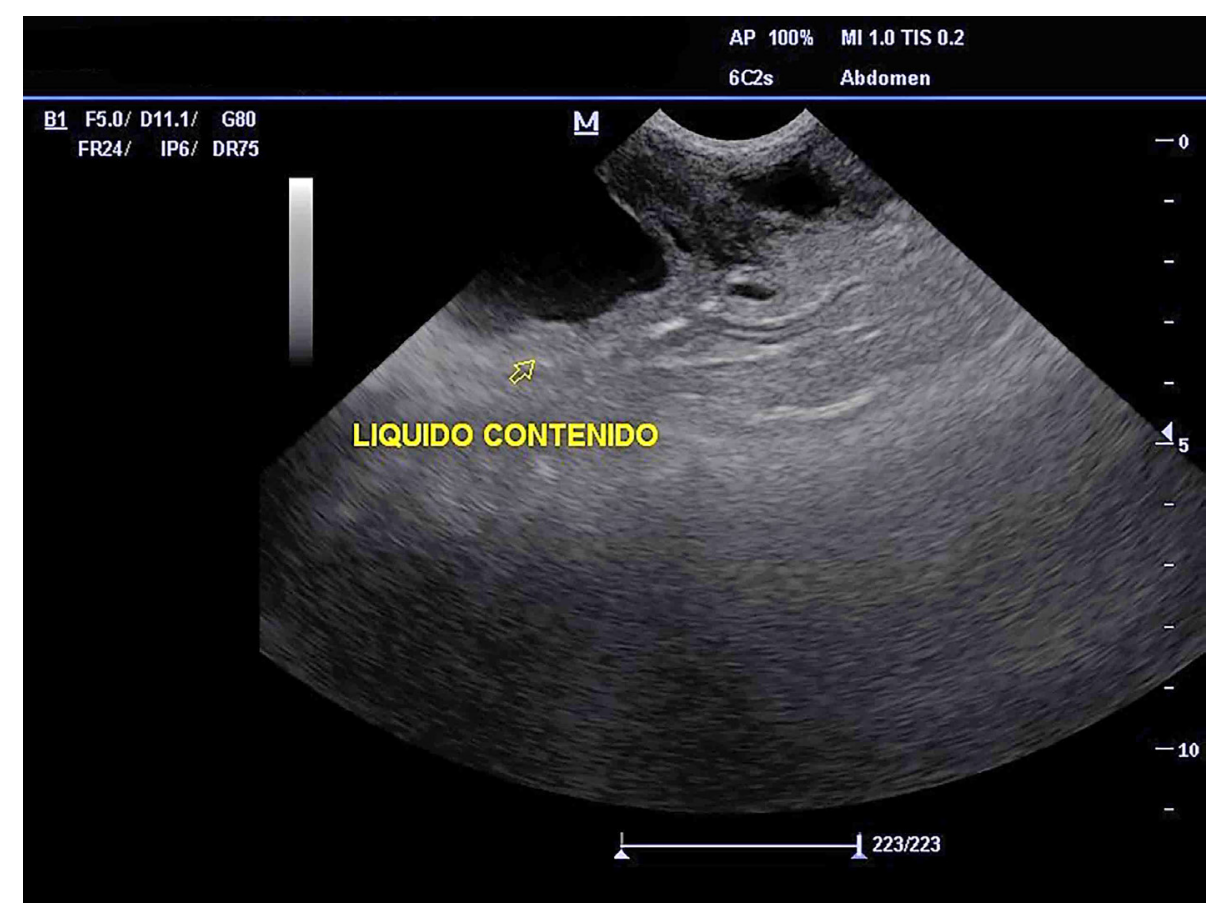

Figura 3. Ultrasonido de abdomen. Se observa el útero con contenido líquido. 
El segundo día de hospitalización, debido al estado de la paciente, se decidió realizar laparotomía para retirar el útero. Para la anestesia quirúrgica se empleó medetomidina vía subcutánea, $0.5 \mathrm{~m} / \mathrm{kg}$ (Dorbene vet, Zoetis, México) y ketamina vía intravenosa, $5 \mathrm{mg} / \mathrm{kg}$ (Anesket, Pisa Agropecuaria, SA de CV). ${ }^{3}$ La medicación fue la misma que el primer día y se añadió terapia de fluidos con lactato de Ringer vía intravenosa, $10 \mathrm{~mL} / \mathrm{kg} / \mathrm{h}$ (Lactato Ringer Vet Braun, B. Braun Vetcare).

Al incidir la cavidad abdominal, solo el cuerno uterino derecho estaba considerablemente aumentado de tamaño, lleno de líquido y ocupaba gran parte de la cavidad abdominal. Se retiró el útero, los oviductos y los ovarios. En la inspección de estos órganos, el cuerno uterino derecho era rojo oscuro, estaba torcido de craneal al cérvix, y ligeramente curveado, medía aproximadamente $26 \mathrm{~cm}$ de largo por $8 \mathrm{~cm}$ de ancho (Figura 4). De su interior se colectaron aproximadamente $800 \mathrm{~mL}$ de líquido sanguinolento, y la mucosa endometrial estaba ligeramente corrugada (Figura 5). El cérvix estaba ocluido. El diagnóstico macroscópico fue torsión uterina e hidrometra unilateral derecha severa. El ovario derecho mostró congestión severa. El resto del tracto reproductor no presentó alteraciones macroscópicas significativas.

Desafortunadamente, la coneja murió aproximadamente a las 15 horas después de la cirugía, y durante este periodo, permaneció con el mismo protocolo de tratamiento que se aplicó el segundo día de su hospitalización. La propietaria no autorizó la necropsia.

\section{Pruebas de laboratorio}

Se tomaron secciones de diversas partes del útero, ovarios y oviductos, las cuales se fijaron en formalina al $10 \%$ por $72 \mathrm{~h}$, y fueron procesadas por la técnica histológica de rutina, se cortaron a $3 \mathrm{~m} \mu$ de grosor y se tiñeron con H\&E, para ser observados con el microscopio óptico. En las secciones histológicas del cuerno uterino derecho, se observó que el endometrio estaba muy hemorrágico y necrosado con pérdida de la mucosa y de las glándulas endometriales, probablemente debido a la gran cantidad de líquido. Las pocas glándulas endometriales que se pudieron observar presentaban dilatación discreta. Además, se apreció marcada congestión y edema en todas las capas uterinas. El diagnóstico histopatológico del útero fue emitido como hemorragia y necrosis endometrial grave, difusa y edema transmural grave difuso. (Figura 6).

El ovario y el oviducto derechos se veían muy congestionados y con zonas hemorrágicas, emitiéndose el diagnóstico histopatológico de hemorragia y congestión grave difusa en ambos órganos (Figura 7). En el resto del tracto reproductor, no se apreciaron cambios histológicos significativos.

\section{Discusión y relevancia clínica}

La demanda de conejos como mascotas, cada vez ha sido mayor, por lo que los propietarios de estos animales acuden con mayor frecuencia a las clínicas veterinarias en busca de algún diagnóstico, tratamiento o simplemente para asesorarse acerca del cuidado y alimentación de este tipo de conejos que llegan a vivir varios años. ${ }^{5-6}$ 


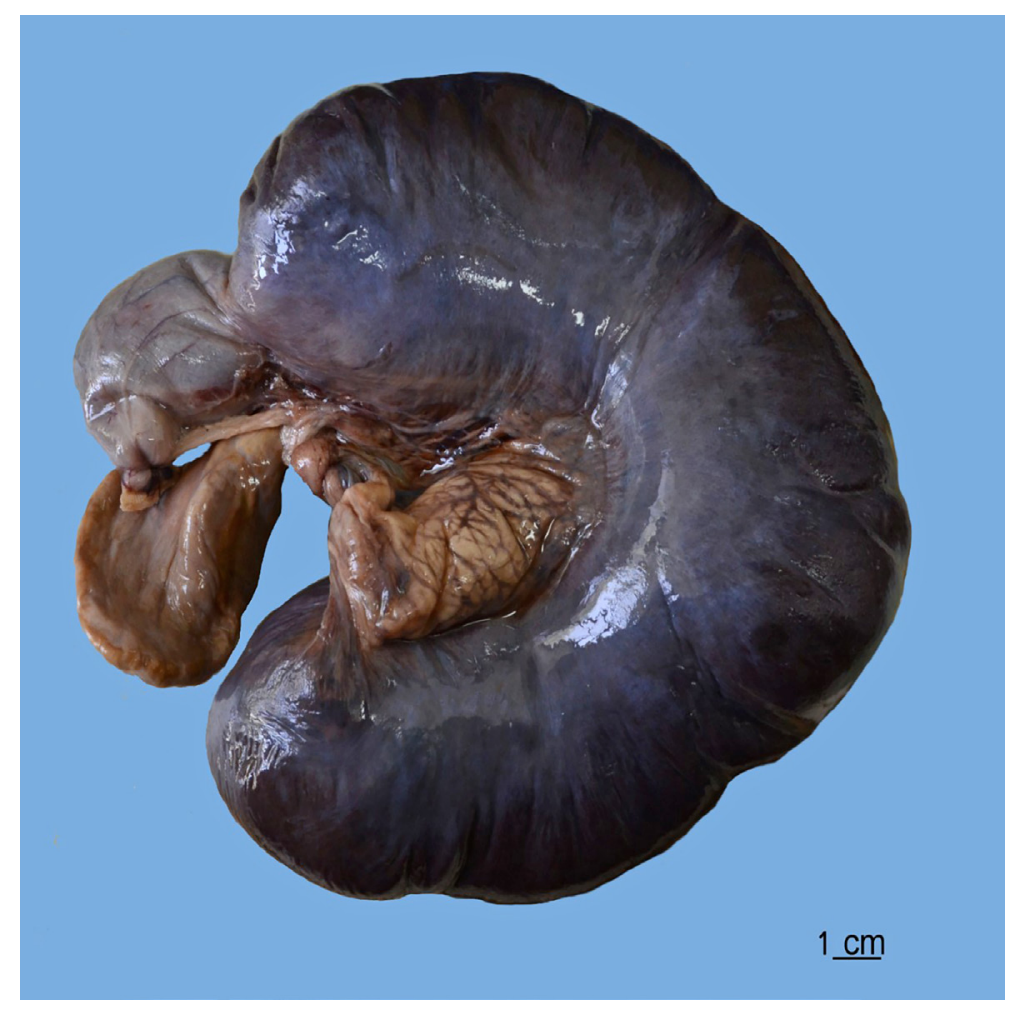

Figura 4. Dilatación del cuerno uterino por acumulo de abundante líquido (hidrometra).

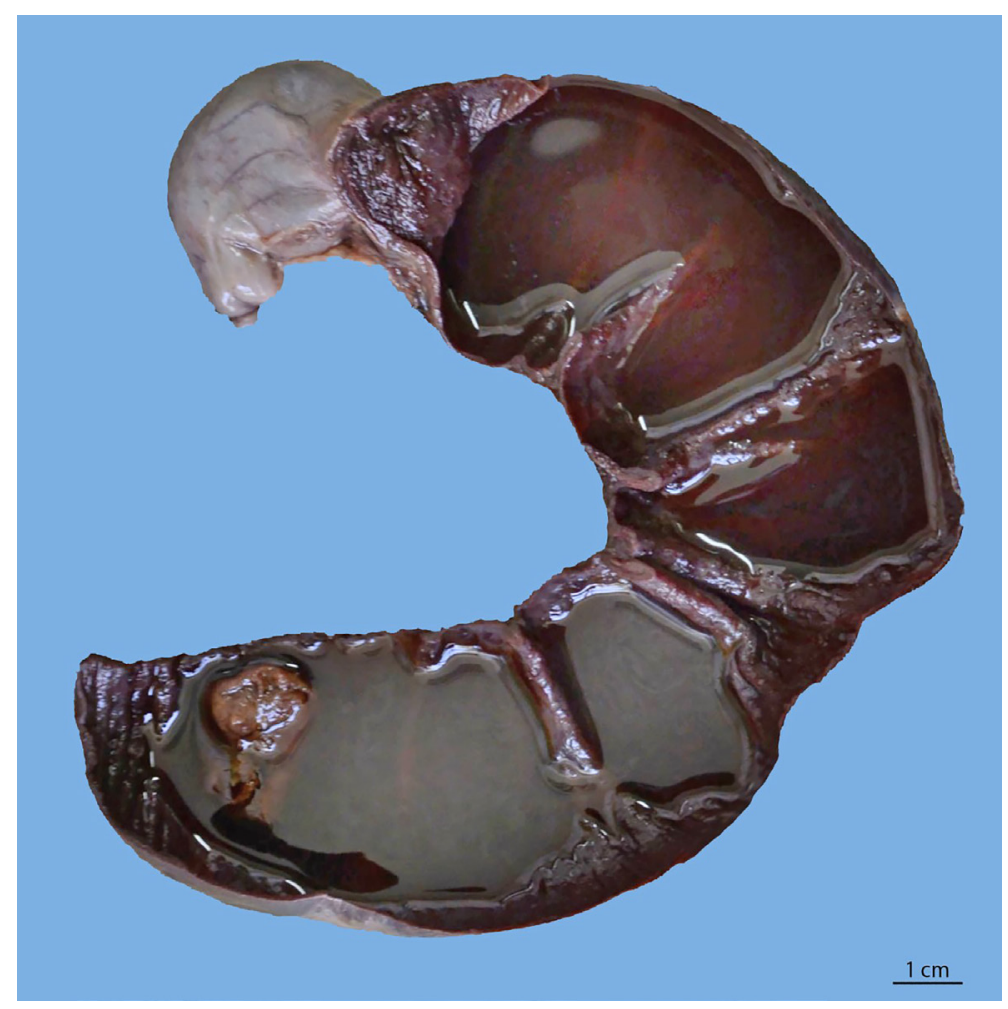

Figura 5. Corte sagital de cuerno uterino con hidrometra. 


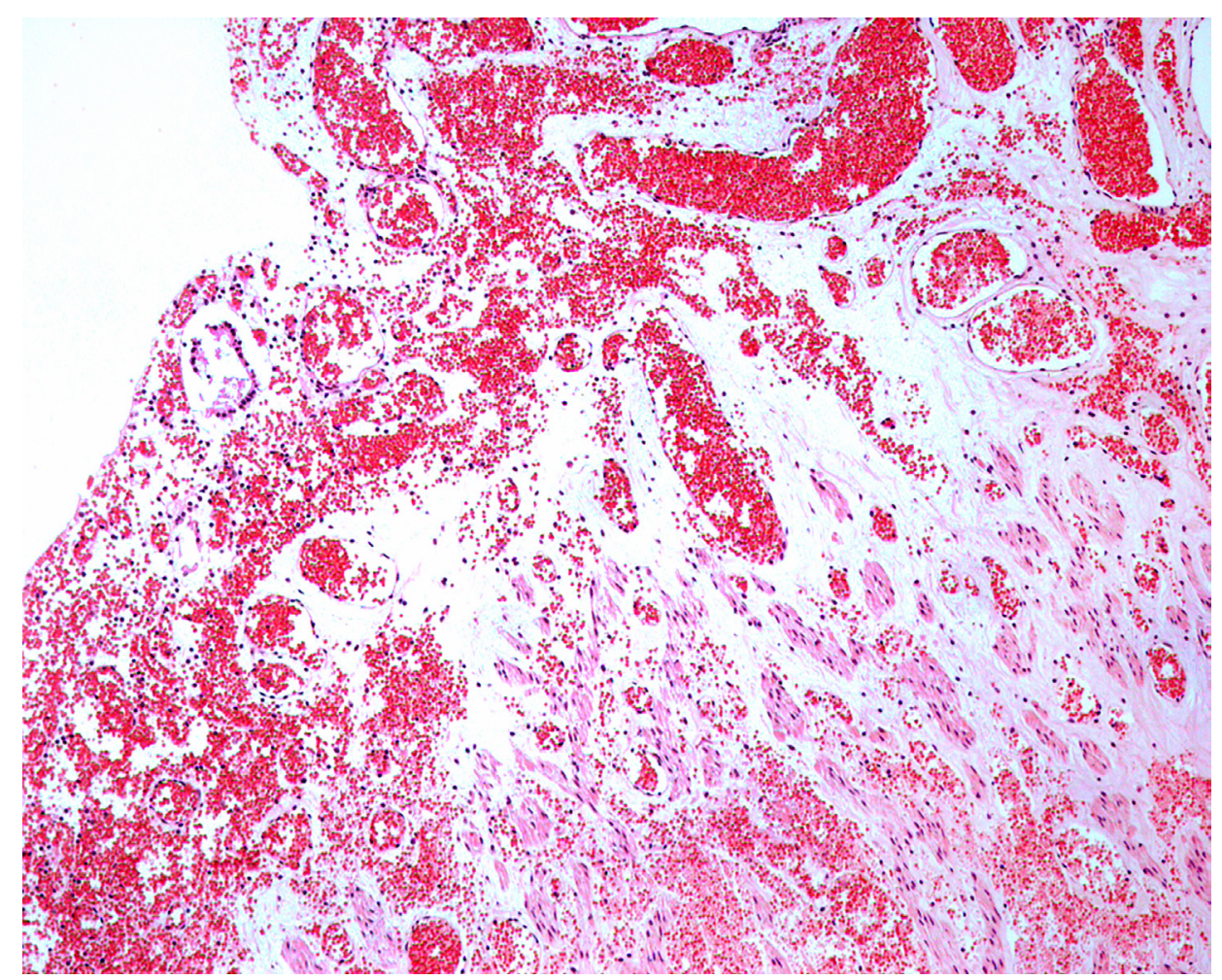

Figura 6. Imagen histológica de útero que muestra glándulas endometriales, y congestión y hemorragia severa con pérdida del epitelio. 10x, H\&E.

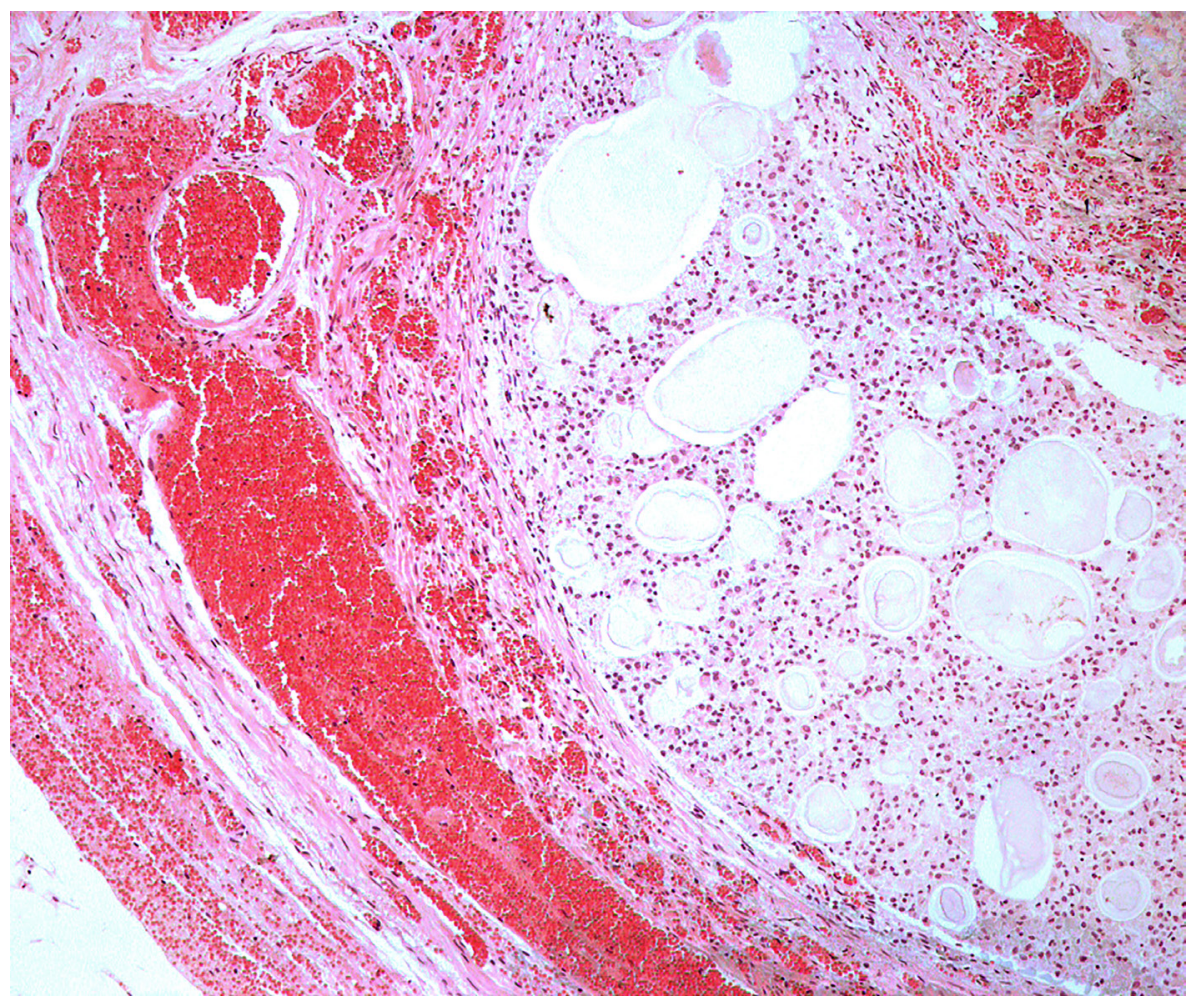

Figura 7. Imagen histológica de ovario que muestra congestión y hemorragia severa. 40x, H\&E. 
Las conejas alcanzan su madurez sexual entre los cuatro y nueve meses de edad. El celo se presenta cada 14 a 16 días con una duración de entre 24 y 36 horas. ${ }^{6}$ La ovulación es inducida y se presenta a las 10 horas postcoito, aunque esta también puede ser inducida mecánicamente por manejo de la hembra con estimulación de la vagina, por la presencia de un macho entero en las proximidades del alojamiento de la hembra, o porque es montada por otra coneja. ${ }^{5-7}$ Si tras la ovulación los óvulos no son fecundados y no hay gestación, se puede presentar un periodo de pseudogestación caracterizado por la presencia de cuerpo lúteo funcional productor de progesterona, lo que induce a desarrollo uterino y mamario igual que en una hembra gestante. ${ }^{5-7}$

Por otro lado, la coneja posee dos cuernos uterinos y dos cérvix independientes, que desembocan en una vagina, por lo que podría presentarse alguna alteración de solo un cuerno uterino como en el caso de la hidrometra. ${ }^{8}$

Al parecer, la incidencia de desórdenes uterinos en conejas de compañía es alta. Al respecto, en un estudio realizado en 50 conejas de compañía con patologías uterinas, se encontró que el adenocarcinoma fue la lesión más frecuente con el $54 \%$ de los casos, seguida de la hiperplasia quística endometrial con $26 \% .{ }^{9}$ En un estudio similar contemplando el tracto reproductor de 59 conejas con desórdenes uterinos, se informó que el 40.6 \% tuvieron hiperplasia quística endometrial y el $30.5 \%$ adenocarcinoma. ${ }^{6}$ En otro estudio realizado en Pensilvania, EUA, se informó que también el adenocarcinoma uterino además de la piometra y la metritis asociada a Pasteurella multocida, fueron patologías comunes para esta especie. ${ }^{10}$ La hidrometra o mucometra han sido descritas con cierta frecuencia en conejas. ${ }^{5,8,10-14}$

La patogenia de la hidrometra en esta especie no está bien esclarecida; sin embargo, este origen se ha informado en hembras nulíparas maduras (mayores de un año), con pseudopreñez después de una auto-ovulación con la producción de progesterona (durante periodos de entre 28 a 33 días, lo que duraría la gestación), la cual estimula la producción de las glándulas endometriales. ${ }^{1-12}$

La hidrometra o mucometra en conejas, perras y gatas, también puede acompañarse de hiperplasia endometrial provocada por la presencia de quistes o tumores ováricos productores de estrógenos o adenocarcinoma endometrial. ${ }^{13-14}$ Estas acumulaciones también pueden ser provocadas por la obstrucción del lumen uterino a consecuencia de aplasia segmental, estenosis o lesiones obstructivas en el cérvix o la vagina por neoplasias como fibromas o leiomiomas entre otras. ${ }^{15}$

Por otro lado, la torsión uterina es rara en conejas; se ha asociado a endometritis, útero grávido en la última semana de gestación y a hidrometra, como en este caso de estudio. ${ }^{10-11}$ La patogenia de la hidrometra y la torsión uterina unilateral en este estudio es incierta, es decir, no se sabe si la hidrometra favoreció a la torsión del cuerno uterino o la torsión con obstrucción de la luz uterina fue un factor predisponente para la hidrometra. Es posible que la coneja haya presentado psedogestaciones y como consecuencia hiperplasia endometrial con hidrometra, que provocó distención y la torsión del cuerno uterino. ${ }^{12}$

Una vez producida la torsión, los vasos venosos se congestionan y el flujo sanguíneo del órgano se interrumpe provocando isquemia tisular y necrosis, esto se detectó en el estudio histológico. Aunque en esta histología se observaron algunas glándulas endometriales dilatadas, la hiperplasia endometrial no se pudo confirmar debido a la hemorragia y necrosis grave del endometrio, y a que éste se descamó hacia el lumen uterino ocupado por abundante líquido. 
De acuerdo con los resultados de patología clínica, el hemograma muestra claramente que la coneja cursaba con anemia severa altamente regenerativa, lo que puede asociarse a la hemorragia endometrial severa, y la morfología eritrocítica sugiere un proceso oxidativo. ${ }^{16}$

Según el perfil bioquímico, la hiperazotemia prerrenal se puede asociar a posible deshidratación por falta de consumo de agua o probable insuficiencia renal, ${ }^{16}$ aunque esta no se pudo corroborar porque no se realizó la necropsia. La elevación de AST se asoció a esfuerzo y catabolismo muscular. ${ }^{16}$ La hipoproteinemia e hipoalbuminemia se relacionaron con pérdidas a terceros espacios (hidrometra) y probable insuficiencia renal. ${ }^{16}$ La ictericia leve puede relacionarse con la obstrucción parcial de la vesícula biliar por la presencia de lodo biliar y a la hemólisis ligera observada en el hemograma. ${ }^{16}$ La muerte de la coneja se asocia a deshidratación y anemia severa, así como a un desequilibrio electrolítico no compensado tras la cirugía debido a lo avanzado de su condición.

Las enfermedades del tracto reproductor en conejas de edad avanzada y no esterilizadas como el adenocarcinoma uterino y la hiperplasia endometrial son comunes. Estas patologías pueden acompañarse de hidrometra y hemometra afectando a ambos cuernos uterinos. ${ }^{13-15}$ El hallazgo más relevante en este caso es que la torsión e hidrometra de un solo cuerno uterino se presentaron juntas, esto significa que quizá están relacionadas, hecho que ha sido poco documentado. Para confirmar el diagnóstico, se recomienda realizar estudios de imagen como radiografías y ultrasonido, lo que sí se hizo en esta coneja. Se sugiere considerar la laparotomía exploratoria en el diagnóstico ante mortem.

Tanto la hidrometra como la torsión uterina en conejas se pueden prevenir con ovariohisterectomía a temprana edad (a partir de los cinco meses de edad aproximadamente). ${ }^{7}$ Cuando una coneja tiene distención abdominal, además de considerar patologías uterinas como en este caso de estudio, se debe descartar enfermedad cardiaca, renal o alguna neoplasia abdominal.

\section{Financiamiento}

Las pruebas de laboratorio, imagen y estudio anatomopatológico fueron financiadas por la propietaria de la paciente.

\section{Agradecimientos}

Agradecemos a Magda Cristina Rico Tavera por la elaboración e interpretación de las pruebas de patología clínica, a Mónica Sampeiro Gama por su participación en las pruebas de imagen y a Jaime Eugenio Córdova López por su colaboración en la edición del material fotográfico.

\section{Conflictos de interés}

Los autores de este trabajo declaran no tener conflictos de intereses.

\section{Contribución de los autores}

EMS supervisó e interpretó los hallazgos anatomopatológicos y redactó el manuscrito.

FPR y MDQP procesaron las muestras e interpretaron los hallazgos anatomopatológicos.

JCG fue el clínico responsable de la paciente. 


\section{Referencias}

1. Leineweber C, Müller E, Marschang RE. Blood reference intervals for rabbits (Oryctolagus cuniculus) from routine diagnostic samples. Tierarztl Prax Ausg K Kleintiere Heimtiere. 2018;46(6):393-8. doi: 10.1055/s-0038-1677403.

2. Hein J, Hartmann K. Reference ranges for laboratory parameters in rabbits. Tierarztl Prax Ausg K Kleintiere Heimtiere. 2003;31(5):321-8. Disponible en: https://www.researchgate.net/publication/297909859_Reference_ranges_ for_laboratory_parameters_in_rabbits

3. Ivey E, Morrisey J. Therapeutics for rabbits. Vet Clin North Am Exot Anim Pract. 2000; 3(1):183-220. doi: https:// doi.org/10.1016/S109-9194(17)30101-9

4. Barter SL. Rabbit Analgesia. Vet Clin Exot Anim. 2011;46(6)14:93-104. doi:10.1016/j.cvex.2010.09.003.

5. Ríos AM, Nadeu CB, García MA, Barceló AM. Patologías del aparato reproductor en conejas (Oryctolagus cuniculus). Profesión Vet. 2007;16(67):87-90.Disponible en: http://www.colvema.org/PDF/conejas.pdf

6. Walter B, Poth T, Böhmer E, Braun J, Matis U. Uterine disorders in 59 rabbits. Vet Rec. 2010;166:230-3. doi: 10.1136/vr.b4749.

7. Varga M. Textbook of rabbit medicine. En: Rabbit basic science, Chapter 1. 2nd ed._Woburn, MA (US): Butterworth-Heinemann. Elsevier; 2013.

8. Barthold WS, Griffey MS, Percy HD. Pathology of laboratory rodenst and rabbits. 4th ed. Ames, lowa (US): Wiley-Blackwell; 2016.

9. Künzel F, Grinninger P, Shibly S, Hassan J, Tichy A, Berghold P, Fuchs-Baumgartinger A. Uterine disorders in 50 pet rabbits. J Am Anim Hosp Assoc. 2015;51:8-14. doi: 10.5326/JAAHA-MS-5812.

10. Harcout-Brown FM. Disorders of the reproductive tract of rabbits. Vet Clin Exot Anim. 2017;20:555-87. doi: http://dx.doi.org/10.1016/j.cvex.2016.11.010

11. Hobbs BA, Parker RF. Uterine torsion associated with either hydrometra or endometritis in two rabbits. Lab Anim Sci. 1990;40(5):535-6. Disponible en: https://www.ncbi.nlm.nih.gov/pubmed/2170759

12. Bray MV, Gaertner DJ, Brownstein DG, Moody KD. Hydrometra in a New Zealand white rabbit. Lab Anim Sci; 1991;41(6):628-9. Disponible en: https:// www.ncbi.nlm.nih.gov/pubmed/1667213

13. Hristov K, Mehandzhiyski N, Peev II, Georgiev G. Case study of hydrometra and uterine adenocarcinoma in a pet rabbit. Medlnform. 2017;(1):544-50. doi: 10.18044/Medinform.201741.544.

14. Polat $B$, Salmanağlu MR. Hydrometra and endometrial hyperplasia in a cat with follicular cyst. J Appl Biol Sci. 2007;1(3):109-10. Disponible en: http://dergipark.gov.tr/download/article-file/414890

15. Sladakovic I, Guzman D, Petritz AO, Mohr CF, McGraw NS. Unilateral cervical and segmental uterine horn aplasia with endometrial hyperplasia, mucometra, and endometritis in a domestic rabbit (Oryctolagus cuniculus). J Exot Pet Med. 2014;24(1):98-104. doi: doi.org/10.1053/j.jepm.2014.11.004.

16. Benson KG, Paul-Murphy J. Clinical pathology of the domestic rabbit: acquisition and interpretation of samples. Vet Clin North Am Exot Anim Pract. 1999;2(3):539-51. doi: doi.org/10.1016/S1094-9194(17)30109-3. 